\title{
OPERATORS WITH AN INTEGRAL REPRSENTATION
}

\author{
RAFFAELLA CILIA AND JOAQUÍN M. GUTIÉRREZ
}

(Communicated by Thomas Schlumprecht)

\begin{abstract}
We introduce a fairly large class of bounded linear operators between Banach spaces which admit an integral representation. It turns out that an operator belongs to this class if and only if it factors through a $C(K)$ space. As an application, we characterize Banach spaces containing no copy of $c_{0}, \mathrm{Ba}$ nach spaces containing no complemented copy of $\ell_{1}$, Grothendieck spaces, and $\mathcal{L}_{\infty}$-spaces. We also study $C(K)$-factorization and extension properties of absolutely continuous operators, giving a partial answer to a question raised in 1985 by H. Jarchow and U. Matter.
\end{abstract}

\section{INTRODUCTION}

We recall some simple notions from measure theory. Let $\Omega$ be a set and $\mathcal{F}$ be a field (also called a Boolean algebra) of subsets of $\Omega$ [11, III, Definition 1.3]. Given a Banach space $X$, let $\mathcal{G}: \mathcal{F} \rightarrow X$ be a vector measure [10, Definition I.1.1]. The variation of $\mathcal{G}$ is the extended nonnegative function $|\mathcal{G}|$ whose value on a set $M \in \mathcal{F}$ is given by

$$
|\mathcal{G}|(M):=\sup _{\pi} \sum_{A \in \pi}\|\mathcal{G}(A)\|
$$

where the supremum is taken over all partitions $\pi$ of $M$ into a finite number of pairwise disjoint members of $\mathcal{F}$.

The semivariation of $\mathcal{G}$ is the extended nonnegative function $\|\mathcal{G}\|$ whose value on a set $M \in \mathcal{F}$ is given by

$$
\|\mathcal{G}\|(M):=\sup \left\{\left|x^{*} \circ \mathcal{G}\right|(M): x^{*} \in X^{*},\left\|x^{*}\right\| \leq 1\right\},
$$

where $\left|x^{*} \circ \mathcal{G}\right|$ is the variation of the scalar-valued measure $x^{*} \circ \mathcal{G}$ [10, Definition I.1.4].

Our starting point is the following well-known result [10, Theorem VI.2.1]:

Theorem 1.1. Given a compact Hausdorff space $K$, a Banach space $X$, and a (bounded linear) operator $T: C(K) \rightarrow X$, there exists a weak-star countably additive measure $\mathcal{G}$ defined on the Borel sets of $K$ with values in $X^{* *}$ such that

(a) $\left\langle x^{*}, \mathcal{G}(\cdot)\right\rangle$ is a regular countably additive Borel measure for each $x^{*} \in X^{*}$;

(b) the operator $X^{*} \rightarrow C(K)^{*}$ given by $x^{*} \mapsto\left\langle x^{*}, \mathcal{G}(\cdot)\right\rangle$ is continuous when both spaces are endowed with the weak-star topology;

(c) $x^{*}(T(f))=\int_{K} f d\left(x^{*} \mathcal{G}\right)$ for each $f \in C(K)$ and each $x^{*} \in X^{*}$;

(d) $\|T\|=\|\mathcal{G}\|(K)$ where $\|\mathcal{G}\|$ denotes the semivariation of $\mathcal{G}$.

Received by the editors August 31, 2015 and, in revised form, February 19, 2016.

2010 Mathematics Subject Classification. Primary 47B10; Secondary 47L20, 46B28, 46 B03.

Key words and phrases. Integral representation, $\infty$-integral operators, extendible operators, factorization through $C(K)$ spaces, absolutely continuous operators.

Both authors were supported in part by Dirección General de Investigación, MTM2015-65825-P Spain. 
Conversely, if $\mathcal{G}$ is an $X^{* *}$-valued vector measure defined on the Borel sets of $K$ for which (a) and (b) hold, then (c) defines an operator from $C(K)$ into $X$ which satisfies (d).

By $\mathcal{L}(E, F)$ we denote the space of all (bounded linear) operators from a Banach space $E$ into a Banach space $F$, endowed with the supremum norm. Note that Theorem 1.1 identifies the operators $T \in \mathcal{L}(C(K), X)$ with the $X^{* *}$-valued measures on the Borel sets of $K$ satisfying conditions (a) and (b).

Theorem 1.1 gave us the idea of considering operators $T \in \mathcal{L}(E, F)$ between arbitrary Banach spaces which may be given an integral representation as shall be defined in Section 2 with respect to a measure that has the properties of $\mathcal{G}$ in Theorem 1.1. The class of all such operators will be denoted by $\mathcal{L}_{\text {ir }}(E, F)$. We endow this class with a complete norm, and prove that an operator belongs to $\mathcal{L}_{\text {ir }}$ if and only if it factors through a $C(K)$ space. The operators that factor through a $C(K)$ space were mentioned in $[19,19.3 .11]$ under the name of strongly $\infty$-factorable operators but, as far as we know, have not received further attention.

Recall that an operator $T \in \mathcal{L}(E, F)$ is weakly compact if it takes the unit ball of $E$ into a relatively weakly compact subset of $F$, and $T$ is said to be completely continuous if it takes weakly null sequences of $E$ into norm null sequences in $F$.

There exists a vast literature on integral operators. To avoid confusion, our operators in $\mathcal{L}_{\text {ir }}$ shouldn't be called integral but rather "operators that admit an integral representation".

To underline the difference between the existing classes of integral operators and $\mathcal{L}_{\text {ir }}$, let us recall that, for $1 \leq p \leq \infty$, an operator $T \in \mathcal{L}(E, F)$ is said to be strictly $p$-integral (sometimes called Pietsch p-integral) if there are a probability measure $\mu$ and operators $U \in \mathcal{L}\left(E, L_{\infty}(\mu)\right)$ and $V \in \mathcal{L}\left(L_{p}(\mu), F\right)$ such that the following diagram commutes:

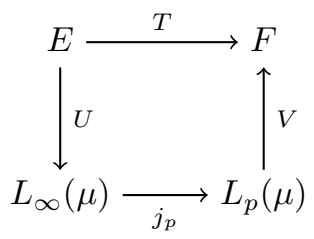

where $j_{p}: L_{\infty}(\mu) \rightarrow L_{p}(\mu)$ is the formal identity. We denote by $\mathcal{S I}_{p}(E, F)$ the class of strictly $p$-integral operators from $E$ into $F$, which is studied for instance in [9, page 97] (more generally in [9, Chapter 5]), in many places of [7], in [19, 19.2.15] under a slightly different name, in [20, page 65], etc. The Pietsch integral operators of [10, Definition VI.3.8] are just the strictly 1-integral operators.

Since every strictly $p$-integral operator factors through a $C(K)$ space, it belongs to $\mathcal{L}_{\text {ir }}$. Moreover, for $p$ finite, every strictly $p$-integral operator is weakly compact and completely continuous [19, Propositions 19.2.11 and 19.2.12], while the operators in $\mathcal{L}_{\text {ir }}$ need not be so. Hence, in general, $\mathcal{L}_{\text {ir }}$ is much larger than $\mathcal{S} \mathcal{I}_{p}$, even in the case $p=\infty$ which will be considered in Proposition 3.7. Recall that, for $1 \leq p \leq q \leq \infty$, we have $\mathcal{S I}_{p} \subseteq \mathcal{S I}_{q}$.

An operator $T \in \mathcal{L}(E, F)$ is said to be $p$-integral (also called Grothendieck $p$ integral) if $k_{F} \circ T \in \mathcal{S I}_{p}\left(E, F^{* *}\right)$, where $k_{F}: F \hookrightarrow F^{* *}$ is the isometric natural embedding of $F$ into its bidual. The references given above for $\mathcal{S I}_{p}$ are also valid for the class $\mathcal{I}_{p}$ of $p$-integral operators. Clearly, $\mathcal{S I}_{p} \subseteq \mathcal{I}_{p}$. 
In [9, page 121] there are references to discoveries of $p$-integral operators which are not strictly $p$-integral for $p$ finite $\neq 2$.

We do not get into the study of the relationship between $\mathcal{L}_{\text {ir }}$ and $\mathcal{I}_{p}$ but we can say that, for $p$ finite, the class $\mathcal{I}_{p}$ is also small since every operator in $\mathcal{I}_{p}$ is weakly compact and completely continuous [19, Propositions 19.2.11 and 19.2.12]. For $1 \leq p \leq q \leq \infty$, we also have $\mathcal{I}_{p} \subseteq \mathcal{I}_{q}$.

Therefore, the class $\mathcal{L}_{\text {ir }}$ is large but at the same time it shares some of the nice properties of $\mathcal{S} \mathcal{I}_{p}$ and $\mathcal{I}_{p}$ such as extension and factorization properties, at least in a weakened form or under some extra conditions.

Our Section 3 is devoted to giving characterizations of Banach spaces containing no copy of $c_{0}$, Banach spaces containing no complemented copy of $\ell_{1}$, Grothendieck spaces, and $\mathcal{L}_{\infty}$-spaces [3, Definition I.2.1] by comparison of $\mathcal{L}_{\text {ir }}$ with the ideals $\mathcal{W}$ of weakly compact operators, $\mathcal{I}_{\infty}, \mathcal{S} \mathcal{I}_{\infty}$, and the ideal $\mathcal{S I}_{\infty}^{\text {wk }}$ of strictly weakly compactly $\infty$-integral operators. The latter was considered by C. Cardassi [5] (see Definition 3.1 below). We prove that, for all Banach spaces $E$ and $F$, we have

$$
\mathcal{S I}_{\infty}^{\mathrm{wk}}(E, F) \subseteq \mathcal{S I}_{\infty}(E, F) \subseteq \mathcal{L}_{\mathrm{ir}}(E, F) \subseteq \mathcal{I}_{\infty}(E, F)
$$

and that the inclusions are, in general, strict. New $C(K)$-factorization and extension properties of absolutely continuous operators are also obtained (see the definition in Section 3 ).

Throughout, $E, F, X, Y$ denote Banach spaces, $E^{*}$ is the dual of $E$, and $B_{E}$ stands for its closed unit ball. The dual ball $B_{E^{*}}$ will always be endowed with the weak-star topology. By $\mathbb{K}$ we represent the scalar field (real or complex). We use the symbol $\mathcal{K}(E, F)$ (respectively, $\mathcal{W}(E, F)$ ) for the space of all compact (respectively, weakly compact) operators. Given $T \in \mathcal{L}(E, F)$, its adjoint is denoted by $T^{*}$.

We say that $T \in \mathcal{L}(E, F)$ is an embedding if $T$ is an isomorphism onto its image $T(E)$. The isometric embedding $h_{E}: E \hookrightarrow C\left(B_{E^{*}}\right)$ is given by $h_{E}(x)\left(x^{*}\right):=x^{*}(x)$ for all $x \in E$ and $x^{*} \in B_{E^{*}}$. By $I_{E}$ we denote the identity map on $E$. The notation $X \equiv Y$ stands for $X$ and $Y$ being isometrically isomorphic.

We say that an operator $T \in \mathcal{L}(E, F)$ is extendible if, for every Banach space $X \supseteq E, T$ admits an extension $\bar{T} \in \mathcal{L}(X, F)$.

For definitions and results in Banach space theory, we refer the reader to [8] and [10].

\section{Factorization of operators With an integral REPRESENTAtion}

In this section we introduce the operators which admit an integral representation and study their factorization properties.

Definition 2.1. We say that an operator $T \in \mathcal{L}(E, F)$ admits an integral representation if

$$
T(x)=\int_{B_{E^{*}}} x^{*}(x) d \mathcal{G} \quad(x \in E),
$$

for some $F^{* *}$-valued measure $\mathcal{G}$ defined on the Borel sets of $B_{E^{*}}$ such that conditions (a) and (b) of Theorem 1.1 are verified when we take $K:=B_{E^{*}}$ and $X:=F$.

We denote by $\mathcal{L}_{\text {ir }}(E, F)$ the space of all operators $T \in \mathcal{L}(E, F)$ that admit an integral representation. For every $T \in \mathcal{L}_{\text {ir }}(E, F)$, we define

$$
\|T\|_{\text {ir }}:=\inf \|\mathcal{G}\|\left(B_{E^{*}}\right)
$$


where the infimum is taken over all measures $\mathcal{G}$ satisfying Definition 2.1. The fact that this function is a norm is contained in the proof of Proposition 2.9

The integral used in Definition 2.1 is the elementary Bartle integral of [10, Definition I.1.12].

The strictly $p$-integral operators admit an integral representation [5, Definition I.2] but the vector measure involved in it has stronger properties than ours. In particular, it is countably additive and $F$-valued.

Proposition 2.2. An operator $T \in \mathcal{L}(E, F)$ admits an integral representation if and only if it has an extension $S \in \mathcal{L}\left(C\left(B_{E^{*}}\right), F\right)$.

Proof. If $T \in \mathcal{L}_{\text {ir }}(E, F)$ and $\mathcal{G}$ is as in Definition [2.1, then, by Theorem 1.1, $\mathcal{G}$ defines the operator $S: C\left(B_{E^{*}}\right) \rightarrow F$ given by

$$
S(f):=\int_{B_{E^{*}}} f d \mathcal{G} \quad\left(f \in C\left(B_{E^{*}}\right)\right) .
$$

The diagram

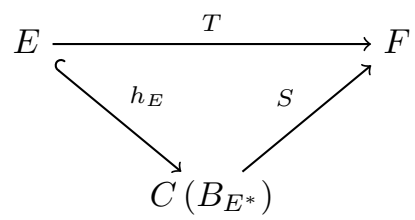

commutes. Indeed, for all $x \in E$, we have

$$
S\left(h_{E}(x)\right)=\int_{B_{E^{*}}} h_{E}(x)\left(x^{*}\right) d \mathcal{G}=\int_{B_{E^{*}}} x^{*}(x) d \mathcal{G}=T(x) .
$$

Hence, $S$ extends $T$. Conversely, if diagram (2.1) commutes, letting $\mathcal{G}$ be the representing measure of $S$, we obtain

$$
T(x)=S\left(h_{E}(x)\right)=\int_{B_{E^{*}}} h_{E}(x)\left(x^{*}\right) d \mathcal{G}=\int_{B_{E^{*}}} x^{*}(x) d \mathcal{G} \quad(x \in E) .
$$

Hence, $T \in \mathcal{L}_{\text {ir }}(E, F)$.

In the remainder of the section we shall give examples of operators which admit an integral representation and of operators which do not admit such a representation.

The following result will be needed:

Proposition 2.3 ([21, Theorem 2.1]). For every compact Hausdorff space $K$, the Banach space $C(K)$ is complemented in $C\left(B_{C(K)^{*}}\right)$ by a norm one projection.

Proof. We sketch the proof for completeness. It is enough to consider the natural embedding $h_{C(K)}$ and the projection

$$
\pi: C\left(B_{C(K)^{*}}\right) \longrightarrow C(K)
$$

defined by

$$
\pi(g)(t):=g\left(\delta_{t}\right) \quad \text { for all } g \in C\left(B_{C(K)^{*}}\right) \text { and } t \in K
$$

where $\delta_{t}$ is an evaluation at $t \in K$.

Corollary 2.4. Every operator $T \in \mathcal{L}(C(K), F)$ admits an integral representation. 
Proof. Factor $T=T \circ I_{C(K)}$ in the form

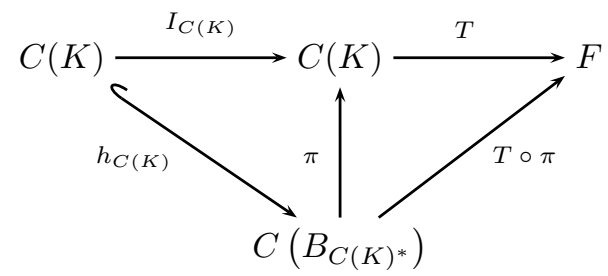

where $\pi$ is as in Proposition 2.3. By Proposition $2.2 T \in \mathcal{L}_{\text {ir }}(E, F)$.

The reader shouldn't think that Corollary 2.4 is trivially contained in Theorem 1.1, since the statement (c) of Theorem 1.1 does not allow, in general, writing

$$
T(f)=\int_{K} f d \mathcal{G} \quad \text { for all } f \in C(K)
$$

because the integral takes values in $F^{* *}$.

We shall now prove that every operator into a $C(K)$ space admits an integral representation and that an operator $T$ belongs to $\mathcal{L}_{\text {ir }}(E, F)$ if and only if $T$ factors through a $C(K)$ space. To this end, we first give a proposition of independent interest, which might be known but we have not found it in the literature. In fact, after submitting our paper we found a related idea in the proof of [4, Theorem 3.4].

Proposition 2.5. For every $T \in \mathcal{L}(E, F)$, there is an operator $\bar{T}: C\left(B_{E^{*}}\right) \rightarrow$ $C\left(B_{F^{*}}\right)$ with $\|\bar{T}\|=\|T\|$ such that the following diagram commutes:

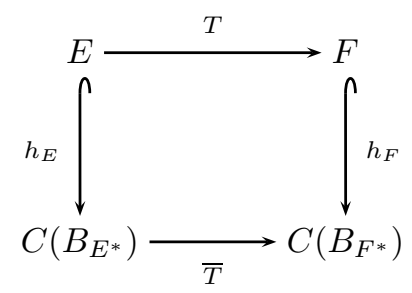

Proof. Assume first that $\|T\|=1$. Define $\bar{T}: C\left(B_{E^{*}}\right) \rightarrow C\left(B_{F^{*}}\right)$ by

$$
\bar{T}(f)\left(y^{*}\right):=f\left(T^{*}\left(y^{*}\right)\right) \quad \text { for all } f \in C\left(B_{E^{*}}\right) \text { and } y^{*} \in B_{F^{*}} .
$$

Then,

$$
\begin{aligned}
\|\bar{T}\| & =\sup _{\|f\| \leq 1}\|\bar{T}(f)\|=\sup _{\|f\| \leq 1} \sup _{y^{*} \in B_{F^{*}}}\left|\bar{T}(f)\left(y^{*}\right)\right| \\
& =\sup _{\|f\| \leq 1} \sup _{y^{*} \in B_{F^{*}}}\left|f\left(T^{*}\left(y^{*}\right)\right)\right| \leq \sup _{\|f\| \leq 1}\|f\|=1 .
\end{aligned}
$$

On the other hand,

$$
\|\bar{T}\| \geq\|\bar{T}(\mathbb{1})\|=1,
$$

hence

$$
\|\bar{T}\|=1=\|T\| .
$$

If $\|T\| \neq 1$, let $U \in \mathcal{L}(E, E)$ and $V \in \mathcal{L}(F, F)$ be given by

$$
U(x):=\frac{x}{\|T\|}, \quad(x \in E) \quad \text { and } \quad V(y):=\|T\| y \quad(y \in F) .
$$


Clearly, $T=V \circ T \circ U$. Moreover,

$$
\|T \circ U\|=\sup _{x \in B_{E}}\|T(U(x))\|=\sup _{x \in B_{E}}\left\|T\left(\frac{x}{\|T\|}\right)\right\|=\frac{1}{\|T\|} \sup _{x \in B_{E}}\|T(x)\|=1 .
$$

Therefore, by the first part of the proof, there is an extension

$$
\overline{T \circ U}: C\left(B_{E^{*}}\right) \rightarrow C\left(B_{F^{*}}\right)
$$

of norm one and such that the left-hand side of diagram (2.2) commutes.

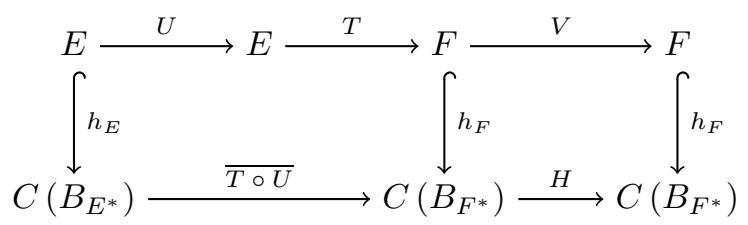

Let $H: C\left(B_{F^{*}}\right) \rightarrow C\left(B_{F^{*}}\right)$ be given by

$$
H(g):=\|T\| g \quad \text { for all } g \in C\left(B_{F^{*}}\right) .
$$

Easily, the right-hand side of diagram (2.2) commutes, so the whole diagram commutes.

We have

$$
\begin{aligned}
\|H \circ \overline{T \circ U}\| & =\sup _{f \in B_{C\left(B_{\left.E^{*}\right)}\right)}}\|H \circ \overline{T \circ U}(f)\| \\
& =\|T\| \sup _{f \in B_{C\left(B_{E^{*}}\right)}}\|\overline{T \circ U}(f)\|=\|T\|\|\overline{T \circ U}\|=\|T\|,
\end{aligned}
$$

and $H \circ \overline{T \circ U}$ is the required operator.

Proposition 2.6. Every operator $T \in \mathcal{L}(E, C(K))$ admits an integral representation.

Proof. It is enough to consider the following diagram:

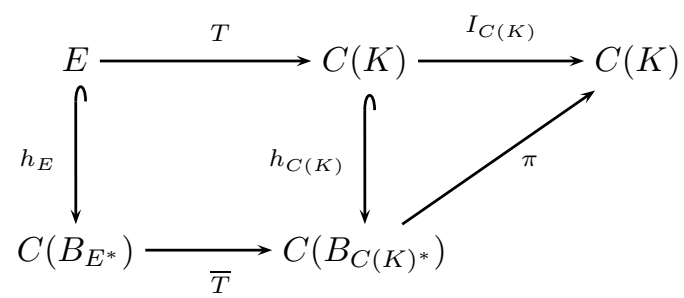

where $\pi$ is the projection of Proposition 2.3 and $\bar{T}$ is the extension constructed in Proposition 2.5 
Theorem 2.7. Given an operator $T \in \mathcal{L}(E, F)$, the following assertions are equivalent:

(a) $T \in \mathcal{L}_{\text {ir }}(E, F)$;

(b) there is an operator $S: C\left(B_{E^{*}}\right) \rightarrow F$ such that $T$ factors as in diagram (2.1);

(c) there are a compact Hausdorff space $K$, an embedding $h^{\prime} \in \mathcal{L}(E, C(K))$, and an operator $S^{\prime} \in \mathcal{L}(C(K), F)$ such that the following diagram is commutative:

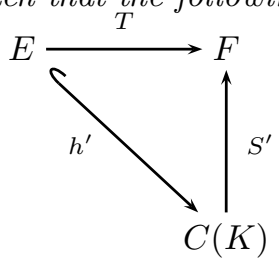

If one (and then all) of these assertions holds, we have

$$
\|T\|_{\text {ir }}=\inf \|S\|=\inf \left\|h^{\prime}\right\|\left\|S^{\prime}\right\|
$$

where the infima are taken over all factorizations as in (b) or (c) respectively.

Proof. (a) $\Leftrightarrow$ (b) is contained in Proposition 2.2 .

(b) $\Rightarrow$ (c) is obvious.

(c) $\Rightarrow$ (b). By Proposition 2.6, $h^{\prime}$ admits an integral representation so, by Proposition 2.2, there is an operator $U: C\left(B_{E^{*}}\right) \rightarrow C(K)$ such that the following diagram commutes:

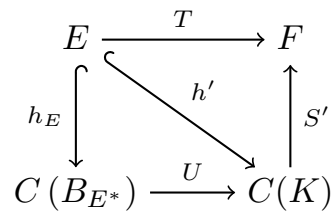

Letting $S:=S^{\prime} \circ U,(\mathrm{~b})$ is proved.

Let us prove the equalities of the norms. Given $\epsilon>0$ and $T \in \mathcal{L}_{\text {ir }}(E, F)$, we can find a measure $\mathcal{G}$ such that

$$
T(x)=\int_{B_{E^{*}}} x^{*}(x) d \mathcal{G} \quad(x \in E)
$$

and

$$
\|\mathcal{G}\|\left(B_{E^{*}}\right)<\|T\|_{\text {ir }}+\epsilon .
$$

Let $S: C\left(B_{E^{*}}\right) \rightarrow F$ be the operator associated with $\mathcal{G}$ by Theorem 1.1. Then $T$ factors as in (b) through the operator $S$ and

$$
\|S\|=\|\mathcal{G}\|\left(B_{E^{*}}\right)<\|T\|_{\text {ir }}+\epsilon .
$$

On the other hand, for every factorization $T=S \circ h_{E}$, if $\mathcal{G}$ is the representing measure of $S$, we obtain

$$
T(x)=S\left(h_{E}(x)\right)=\int_{B_{E^{*}}} h_{E}(x)\left(x^{*}\right) d \mathcal{G}=\int_{B_{E^{*}}} x^{*}(x) d \mathcal{G} \quad(x \in E),
$$

so $T \in \mathcal{L}_{\text {ir }}(E, F)$ and

$$
\|T\|_{\text {ir }} \leq\|\mathcal{G}\|\left(B_{E^{*}}\right)=\|S\|,
$$


and the equality

is proved.

$$
\|T\|_{\text {ir }}=\inf \|S\|
$$

To obtain the second equality, let $T \in \mathcal{L}_{\text {ir }}(E, F)$. By (b), given $\epsilon>0$, we can find a factorization $T=S \circ h_{E}$ such that

$$
\|S\|<\|T\|_{\text {ir }}+\epsilon .
$$

Letting $K:=B_{E^{*}}, S^{\prime}:=S$, and $h^{\prime}:=h_{E}$, we have

$$
\left\|h^{\prime}\right\|\left\|S^{\prime}\right\|=\left\|h_{E}\right\|\|S\|=\|S\|<\|T\|_{\text {ir }}+\epsilon .
$$

On the other hand, let $T=S^{\prime} \circ h^{\prime}$ be a factorization as in (c). By the proof of Proposition 2.6. we obtain a factorization as in diagram (2.3) where $U=\pi \circ \overline{h^{\prime}}$.

Using inequality (2.4) and Proposition 2.5, we have

$$
\|T\|_{\text {ir }} \leq\left\|S^{\prime} \circ U\right\| \leq\left\|S^{\prime}\right\|\left\|\pi \circ \overline{h^{\prime}}\right\| \leq\left\|S^{\prime}\right\|\|\pi\|\left\|\overline{h^{\prime}}\right\|=\left\|S^{\prime}\right\|\left\|h^{\prime}\right\| .
$$

Therefore,

and this finishes the proof.

$$
\|T\|_{\text {ir }}=\inf \left\|S^{\prime}\right\|\left\|h^{\prime}\right\|
$$

The factorization of Theorem 2.7(c) shouldn't be confused with "similar" factorizations that can be found for instance in [10, Theorem VI.3.11] or [20, page 113] where $T$ is Pietsch integral and $S$ (or $S^{\prime}$ ) is absolutely summing. In Theorem 2.7. the operator $S^{\prime}$ is arbitrary.

For $1 \leq p \leq 2$, every absolutely $p$-summing operator belongs to $\mathcal{L}_{\text {ir }}$, Theorem 2.8 and Corollary 2.16].

When $E$ and $F$ have cotype 2 , the operators in $\mathcal{L}_{\text {ir }}$ are easily characterized:

Corollary 2.8. Let $E$ and $F$ be Banach spaces of cotype 2. For an operator $T \in \mathcal{L}(E, F)$ the following assertions are equivalent:

(a) $T \in \mathcal{L}_{\text {ir }}(E, F)$;

(b) $T$ is absolutely $p$-summing for some (and then all) $1 \leq p<\infty$.

Proof. (a) $\Rightarrow$ (b) since every operator from a $C(K)$ space into a cotype 2 space is absolutely 2-summing [9, Theorem 11.14].

(b) $\Rightarrow$ (a). If $T$ is absolutely 2 -summing, then it factors through a $C(K)$ space [9, Corollary 2.16].

For the equivalence between 2-summing and $p$-summing operators, see [9, Corollary 11.16].

The following result can be proved in a standard way using [7, Criterion 9.4] and our Proposition 2.5 and Theorem 2.7.

Proposition 2.9. The pair $\left(\mathcal{L}_{\mathrm{ir}},\|\cdot\|_{\mathrm{ir}}\right)$ is a Banach operator ideal.

Corollary 2.10. For every $T \in \mathcal{L}(E, C(K))$, we have

$$
\|T\|_{\text {ir }}=\|T\| .
$$

Proof. From Proposition 2.6 and inequality (2.4), we have

$$
\|T\|_{\text {ir }} \leq\|\pi \circ \bar{T}\| \leq\|\pi\|\|\bar{T}\|=\|\bar{T}\|=\|T\| .
$$

Since the reverse inequality is always true [7, Proposition 9.3], we obtain the result. 
Corollary 2.11. For every $T \in \mathcal{L}(C(K), F)$, we have $\|T\|_{\text {ir }}=\|T\|$.

Proof. From Corollary 2.4, we have $\|T\|_{\text {ir }} \leq\|T\|\|\pi\|=\|T\|$. The reverse inequality is always true [7, Proposition 9.3].

Corollary 2.12. An operator $T \in \mathcal{L}(E, F)$ belongs to $\mathcal{L}_{\mathrm{ir}}(E, F)$ if and only if there are a $C(K)$ space and operators $U \in \mathcal{L}(E, C(K))$ and $V \in \mathcal{L}(C(K), F)$ such that $T=V \circ U$. Moreover,

$$
\|T\|_{\text {ir }}=\inf \|U\|\|V\|,
$$

where the infimum is taken over all possible factorizations of that form.

Proof. Use Theorem 2.7. Corollary 2.4, and Proposition 2.6. For the equality of the norms, use Corollaries 2.10 and 2.11, and the ideal property contained in Proposition 2.9

\section{Characterizations of Banach spaces in terms of operators WITH AN INTEGRAL REPRESENTATION}

As stated in the Introduction, this section is devoted to giving applications of the ideal $\mathcal{L}_{\text {ir }}$ to characterize Banach spaces containing no copy of $c_{0}$, Banach spaces containing no complemented copy of $\ell_{1}$, Grothendieck spaces, and $\mathcal{L}_{\infty}$-spaces.

Definition 3.1. We say that an operator $T \in \mathcal{L}(E, F)$ is strictly weakly compactly $\infty$-integral if it admits a factorization as in diagram (2.1) with $S$ a weakly compact operator. By $\mathcal{S I}_{\infty}^{\mathrm{wk}}(E, F)$ we denote the space of all strictly weakly compactly $\infty$-integral operators from $E$ into $F$. Given $T \in \mathcal{S I}_{\infty}^{\text {wk }}(E, F)$, its strictly weakly compactly $\infty$-integral norm is defined by inf $\|S\|$, where the infimum is taken over all factorizations as in diagram (2.1) with $S$ weakly compact (see the last line of [5. Theorem I.8]).

This class has been studied in [5, Theorem I.8] under a different name.

Obviously $\mathcal{S I}_{\infty}^{\text {wk }}(E, F) \subseteq \mathcal{S} \mathcal{I}_{\infty}(E, F)$. The natural inclusion of $C[0,1]$ into the space $L_{\infty}[0,1]$ is an operator in $\mathcal{S} \mathcal{I}_{\infty}$ which is not in $\mathcal{S I}_{\infty}^{\text {wk }}$.

We start by giving characterizations of Banach spaces $F$ containing no copy of $c_{0}$.

Theorem 3.2. Given a Banach space $F$, the following assertions are equivalent:

(a) F contains no copy of $c_{0}$;

(b) for every Banach space $E, \mathcal{L}_{\mathrm{ir}}(E, F) \equiv \mathcal{S I}_{\infty}^{\mathrm{wk}}(E, F)$;

(c) $\mathcal{L}_{\text {ir }}\left(c_{0}, F\right) \equiv \mathcal{S I}_{\infty}^{\mathrm{wk}}\left(c_{0}, F\right)$;

(d) there is a non-Grothendieck space $E$ such that $\mathcal{L}_{\text {ir }}(E, F) \equiv \mathcal{S I}_{\infty}^{\mathrm{wk}}(E, F)$;

(e) there is a non-Grothendieck space $E$ such that $\mathcal{L}_{\text {ir }}(E, F) \subseteq \mathcal{W}(E, F)$;

(f) there is a Banach space $E$ without the Dunford-Pettis property such that $\mathcal{L}_{\text {ir }}(E, F) \equiv \mathcal{S I}_{\infty}^{\mathrm{wk}}(E, F)$;

(g) there is a Banach space $E$ containing no copy of $\ell_{1}$ such that $\mathcal{L}_{\text {ir }}(E, F) \equiv$ $\mathcal{S I}_{\infty}^{\mathrm{wk}}(E, F)$;

(h) there is a Banach space $E$ with a quotient isomorphic to $c_{0}$ such that

$$
\mathcal{L}_{\text {ir }}(E, F) \equiv \mathcal{S I}_{\infty}^{\mathrm{wk}}(E, F)
$$

Proof. (a) $\Rightarrow$ (b). Suppose that $F$ contains no copy of $c_{0}$ and let $T \in \mathcal{L}_{\text {ir }}(E, F)$. Then $T$ factors as in diagram (2.1). Since $F$ contains no copy of $c_{0}$ and $C\left(B_{E^{*}}\right)$ 
has property (V) [17], $S$ is weakly compact and $T \in \mathcal{S I}_{\infty}^{\mathrm{wk}}(E, F)$. The inclusion $\mathcal{S I}_{\infty}^{\mathrm{wk}}(E, F) \subseteq \mathcal{L}_{\text {ir }}(E, F)$ is obvious. The equality of the norms is clear.

(b) $\Rightarrow$ (c) $\Rightarrow$ (d) $\Rightarrow$ (e), (b) $\Rightarrow$ (f), (c) $\Rightarrow$ (g), and (b) $\Rightarrow$ (h) are obvious.

$(\mathrm{h}) \Rightarrow(\mathrm{d})$ since a Grothendieck space cannot have a quotient isomorphic to $c_{0}$.

$(\mathrm{e}) \Rightarrow$ (a). Let $E$ be the Banach space provided by (e) and assume that $j$ : $c_{0} \hookrightarrow F$ is an embedding. Since $E$ is not Grothendieck, there is an operator $T \in \mathcal{L}\left(E, c_{0}\right) \backslash \mathcal{W}\left(E, c_{0}\right)$. The operator $j \circ T: E \rightarrow F$ factors through $c_{0}$ so it admits an integral representation, but it is not weakly compact, a contradiction.

(f) $\Rightarrow$ (a). Let $E$ be the Banach space provided by (f) and assume that $j: c_{0} \hookrightarrow F$ is an embedding. Since $E$ does not have the Dunford-Pettis property, there is an operator $T \in \mathcal{W}\left(E, c_{0}\right)$ which is not completely continuous [18, Proposition 4]. Then $j \circ T$ is not completely continuous and therefore does not belong to $\mathcal{S I}_{\infty}^{\text {wk }}(E, F)$, but it has an integral representation.

$(\mathrm{g}) \Rightarrow(\mathrm{a})$. Let $E$ be the space provided by (g) and let $T: E \rightarrow c_{0}$ be a noncompact operator [8, Exercise XII.1]. If there is an embedding $j: c_{0} \hookrightarrow F$, then $j \circ T \in \mathcal{L}_{\text {ir }}(E, F)$ so, by $(\mathrm{g}), j \circ T \in \mathcal{S I}_{\infty}^{\mathrm{wk}}(E, F)$. Since $E$ contains no copy of $\ell_{1}$, Rosenthal's $\ell_{1}$-theorem implies that $\mathcal{S I}_{\infty}^{\mathrm{wk}}(E, F) \subseteq \mathcal{K}(E, F)$. Therefore $j \circ T$ is compact, a contradiction.

Remark 3.3. From Theorem 3.2 it follows that $F \nsupseteq c_{0}$ implies $\mathcal{L}_{\text {ir }}(E, F) \subseteq \mathcal{W}(E, F)$ for every Banach space $E$. It is clear that not every weakly compact operator into $F$ admits an integral representation. For instance, if $E=F$ is a reflexive infinitedimensional Banach space, then $I_{E}$ does not admit an integral representation.

The following result gives a characterization of Grothendieck spaces and shows that the equivalence (a) $\Leftrightarrow(\mathrm{e})$ in Theorem 3.2 is best possible.

Proposition 3.4. Given a Banach space E, the following assertions are equivalent:

(a) $E$ is a Grothendieck space;

(b) there is a Banach space $F$ containing $c_{0}$ such that $\mathcal{L}_{\text {ir }}(E, F)=\mathcal{W}(E, F)$;

(c) there is a Banach space $F$ containing $c_{0}$ such that $\mathcal{L}_{\text {ir }}(E, F) \subseteq \mathcal{W}(E, F)$.

Proof. (a) $\Rightarrow$ (b). It is enough to take $F=c_{0}$.

(b) $\Rightarrow$ (c) is obvious.

(c) $\Rightarrow$ (a). Suppose that $E$ is not Grothendieck. Then there is a nonweakly compact operator $T \in \mathcal{L}\left(E, c_{0}\right)$. Let $F$ be the space provided by (c) and let $j$ : $c_{0} \hookrightarrow F$ be an embedding. Then $j \circ T$ is not weakly compact but it has an integral representation, a contradiction.

Recall that the following assertions are equivalent for a Banach space $F$, Theorem V.10]:

(a) $F$ contains a complemented copy of $\ell_{1}$;

(b) $F^{*}$ contains a copy of $c_{0}$;

(c) $F^{*}$ contains a copy of $\ell_{\infty}$.

Theorem 3.5. Given a Banach space $F$, the following assertions are equivalent:

(a) $F$ contains no complemented copy of $\ell_{1}$;

(b) for every Banach space $E, \mathcal{L}_{\mathrm{ir}}\left(E, F^{*}\right) \equiv \mathcal{S I}_{\infty}^{\mathrm{wk}}\left(E, F^{*}\right)$;

(c) there is a nonreflexive Banach space $E$ such that $\mathcal{L}_{\mathrm{ir}}\left(E, F^{*}\right) \equiv \mathcal{S I}_{\infty}^{\mathrm{wk}}\left(E, F^{*}\right)$;

(d) there is a nonreflexive Banach space $E$ such that $\mathcal{L}_{\mathrm{ir}}\left(E, F^{*}\right) \subseteq \mathcal{W}\left(E, F^{*}\right)$. 
Proof. (a) $\Rightarrow(\mathrm{b})$. By the previous comment, it is enough to use part (a) $\Rightarrow$ (b) of Theorem 3.2

(b) $\Rightarrow$ (c) $\Rightarrow$ (d) are obvious.

$(\mathrm{d}) \Rightarrow$ (a). Let $E$ be the space provided by (d). There is an operator $T \in$ $\mathcal{L}\left(E, \ell_{\infty}\right) \backslash \mathcal{W}\left(E, \ell_{\infty}\right)$. Indeed, if $E$ is separable, we choose as $T$ the canonical embedding into $\ell_{\infty}$. If $E$ is not separable, it contains a nonreflexive separable subspace $X$. Let $j: X \hookrightarrow \ell_{\infty}$ be the canonical embedding. By the injectivity of $\ell_{\infty}[9$, Theorem 4.14], we can extend $j$ to an operator $T \in \mathcal{L}\left(E, \ell_{\infty}\right)$ which is clearly not weakly compact.

Assume there is an embedding $j: \ell_{\infty} \hookrightarrow F^{*}$. Clearly, $j \circ T \in \mathcal{L}_{\text {ir }}\left(E, F^{*}\right)$. By (d), $j \circ T \in \mathcal{W}\left(E, F^{*}\right)$, a contradiction.

We say that an operator $T \in \mathcal{L}(E, F)$ is absolutely continuous [16] (see also [9, page 311]) if there are $1 \leq p<+\infty$, a Banach space $G$, an absolutely $p$-summing operator $S \in \mathcal{L}(E, G)$ and, for each $\epsilon>0$, an $N(\epsilon)>0$ such that

$$
\|T(x)\| \leq N(\epsilon)\|S(x)\|+\epsilon\|x\| \quad(x \in E) .
$$

Every absolutely continuous operator is weakly compact [9, Corollary 15.4]. It is well known that, if $E$ is an $\mathcal{L}_{\infty}$-space, then an operator on $E$ is absolutely continuous if and only if it is weakly compact. This is also true for other spaces $E$, such as the disk algebra, which are not $\mathcal{L}_{\infty}$-spaces $[13, \S 3]$. Under the uniform norm, the ideal $\mathcal{A C}$ of absolutely continuous operators is closed and injective [9, Corollary 15.4]. Recall also that every compact operator is absolutely continuous [16, Theorem 2.1].

The following result characterizes $\mathcal{L}_{\infty}$-spaces.

Theorem 3.6. Let $E$ be a Banach space. The following assertions are equivalent:

(a) $E$ is an $\mathcal{L}_{\infty}$-space;

(b) $k_{E} \in \mathcal{L}_{\text {ir }}\left(E, E^{* *}\right)$;

(c) for every Banach space $F$, we have $\mathcal{W}(E, F)=\mathcal{S I}_{\infty}^{\mathrm{wk}}(E, F)$;

(d) for every Banach space $F$, we have $\mathcal{W}(E, F) \subseteq \mathcal{L}_{\text {ir }}(E, F)$;

(e) for every Banach space $F$, we have $\mathcal{A C}(E, F)=\mathcal{S I}_{\infty}^{\mathrm{wk}}(E, F)$;

(f) for every Banach space $F$, we have $\mathcal{A C}(E, F) \subseteq \mathcal{L}_{\text {ir }}(E, F)$;

(g) there is $\lambda \geq 1$ such that, for all Banach spaces $X$ and $F$ with $X \supseteq E$ and every operator $T \in \mathcal{A C}(E, F), T$ admits an extension $\bar{T} \in \mathcal{A C}(X, F)$ with $\|\bar{T}\| \leq \lambda\|T\|$.

Proof. (a) $\Rightarrow$ (b). Since $E$ is an $\mathcal{L}_{\infty}$-space, $E^{* *}$ is injective [3, Proposition 1.33]. In particular, $k_{E}$ admits an extension to $C\left(B_{E^{*}}\right)$. Hence, $k_{E} \in \mathcal{L}_{\text {ir }}\left(E, E^{* *}\right)$.

(b) $\Rightarrow$ (c). By (b), there is an operator $S: C\left(B_{E^{*}}\right) \rightarrow E^{* *}$ such that $k_{E}=S \circ h_{E}$. Let $T \in \mathcal{W}(E, F)$. Then $T^{* *} \in \mathcal{W}\left(E^{* *}, F\right)$, so $T^{* *} \circ S \circ h_{E}=T^{* *} \circ k_{E}=T$ with $T^{* *} \circ S \in \mathcal{W}$ which implies that $T \in \mathcal{S I}_{\infty}^{\mathrm{wk}}(E, F)$.

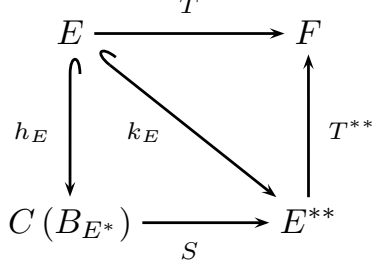

(c) $\Rightarrow(\mathrm{d}) \Rightarrow$ (f) are obvious.

(c) $\Rightarrow$ (e) is clear from the inclusions:

$$
\mathcal{A C}(E, F) \subseteq \mathcal{W}(E, F)=\mathcal{S I}_{\infty}^{\mathrm{wk}}(E, F) \subseteq \mathcal{A C}(E, F)
$$


$(\mathrm{e}) \Rightarrow(\mathrm{g})$. Suppose that (e) holds. Since the identity map from $\mathcal{S I}_{\infty}^{\mathrm{wk}}(E, F)$ onto $\mathcal{A C}(E, F)$ is continuous [7, Proposition 9.3], the open mapping theorem implies that both spaces are isomorphic [15, Corollary 1.6.8], so there is $\alpha>0$ such that the strictly weakly compactly $\infty$-integral norm of $T$ is less than or equal to $\alpha\|T\|$ for every $T \in \mathcal{A C}(E, F)$.

By the definition of the strictly weakly compactly $\infty$-integral norm of $T$ (Definition 3.1), we can find an operator $S \in \mathcal{W}\left(C\left(B_{E^{*}}\right), F\right)$ satisfying diagram (2.1) with $\|S\|<2 \alpha\|T\|$. Let $i: E \hookrightarrow X$ be an embedding. Since $C\left(B_{E^{*}}\right)^{* *}$ is injective, there is $\beta \geq 1$ such that $k_{C} \circ h_{E}$ has an extension $\overline{k_{C} \circ h_{E}} \in \mathcal{L}\left(X, C\left(B_{E^{*}}\right)^{* *}\right)$ of norm $\leq \beta$, where $k_{C}: C\left(B_{E^{*}}\right) \hookrightarrow C\left(B_{E^{*}}\right)^{* *}$ is the natural embedding.

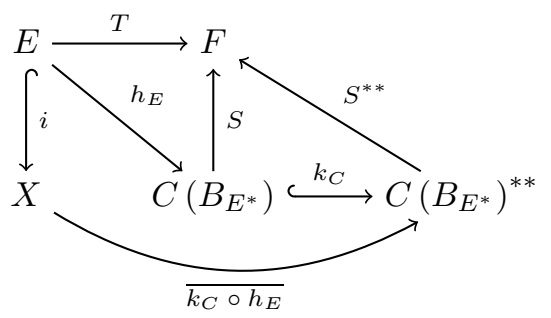

Therefore, $T$ has an extension $S^{* *} \circ \overline{k_{C} \circ h_{E}} \in \mathcal{L}(X, F)$ with norm

$$
\left\|S^{* *} \circ \overline{k_{C} \circ h_{E}}\right\| \leq \beta\|S\|<2 \alpha \beta\|T\| .
$$

Since $S \in \mathcal{A C}$ if and only if $S^{* *} \in \mathcal{A C}$ [9, Corollary 15.5], the above given extension belongs to $\mathcal{A C}(X, F)$ and $(\mathrm{g})$ is proved.

(f) $\Rightarrow$ (g). Let $T \in \mathcal{A C}(E, F)$. Since $T$ is weakly compact, by [6, Corollary 1] (see also [12, Corollary 3.2.3]), there are a reflexive Banach space $G$ and operators $A \in \mathcal{L}(E, G)$ and $B \in \mathcal{L}(G, F)$ such that $T=B \circ A$. Since the ideal $\mathcal{A C}$ is closed and injective, $A$ may be assumed to be absolutely continuous [12, Proposition 5.3.3]. By (f), $A$ factors as in diagram (2.1).

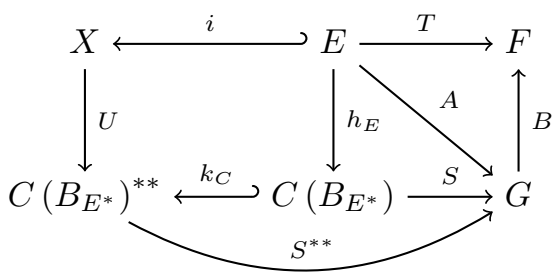

Let $i: E \hookrightarrow X$ be an embedding. By the injectivity of $C\left(B_{E^{*}}\right)^{* *}, k_{C} \circ h_{E}$ has an extension $U: X \rightarrow C\left(B_{E^{*}}\right)^{* *}$. Therefore, $T$ has an extension $B \circ S^{* *} \circ$ $U \in \mathcal{A C}(X, F)$, where we have used the fact that $S^{* *} \in \mathcal{A C}$. The proof of [14, Theorem 2.2] and the Remark following it concerning the control of the norm of the extension may be adapted to the case of absolutely continuous operators thanks to the injectivity of $\mathcal{A C}$.

$(\mathrm{g}) \Rightarrow(\mathrm{a})$. Let $F$ be a finite-dimensional Banach space. Then every operator $T \in \mathcal{L}(E, F)$ is compact and hence absolutely continuous [16, Theorem 2.1]. Let $X \supseteq E$. By (g), $T$ admits an extension $\bar{T} \in \mathcal{L}(X, F)=\mathcal{A C}(X, F)$ with $\|\bar{T}\| \leq \lambda\|T\|$. By [22, Theorem 4.2], $E$ is an $\mathcal{L}_{\infty}$-space. 
The implication (a) $\Rightarrow$ (c) of Theorem 3.6 was essentially known (see [5, Example I.5] for $E=C(K)$; for a generic $\mathcal{L}_{\infty}$-space $E$, use the fact that $E^{* *}$ is complemented in a $C(K)$ space [3, Proposition 1.33]).

The assertions (e), (f), and (g) of Theorem 3.6 give a partial answer to a longstanding open problem about factorization of absolutely continuous operators [13, §7, Problem 1].

In Section 2 we have proved that $T \in \mathcal{L}(E, F)$ belongs to $\mathcal{L}_{\text {ir }}(E, F)$ if and only if $T$ factors through a $C(K)$ space. It is clear that factorization through an $L_{\infty}(\Omega, \mu)$-space, where $(\Omega, \Sigma, \mu)$ is a $\sigma$-finite measure space, is a stronger property as the following well-known result shows.

Proposition 3.7. Given an operator $T \in \mathcal{L}(E, F)$, consider the assertions:

(a) there is an embedding $h: E \hookrightarrow L_{\infty}(\Omega, \mu)$ and an operator $S: L_{\infty}(\Omega, \mu) \rightarrow F$ such that $T=S \circ h$, in other words, $T \in \mathcal{S I}_{\infty}(E, F)$;

(b) $T$ is extendible;

(c) $T \in \mathcal{L}_{\text {ir }}(E, F)$.

Then, $(\mathrm{a}) \Leftrightarrow(\mathrm{b}) \Rightarrow(\mathrm{c})$, but $(\mathrm{c}) \nRightarrow(\mathrm{a})$ in general.

The identity map on $c_{0}$ is an operator satisfying (c) but not (a).

From the above results, we have the following chain of inclusions for all $1 \leq p \leq$ $\infty$ :

$$
\begin{aligned}
\mathcal{S I}_{p}(E, F) & \subseteq\left\{\text { operators factoring through some } L_{\infty}(\Omega, \mu)\right\} \\
& =\{\text { extendible operators }\} \subseteq \mathcal{L}_{\text {ir }}(E, F)
\end{aligned}
$$

and, if $F$ contains no copy of $c_{0}$ and $p=\infty$, all these spaces coincide.

If $E$ is injective, then every operator on $E$ or into $E$ is extendible [22, Proposition 1.1].

The following question seems natural: if an operator $T$ factors as in diagram (2.1) through a weakly compact operator $S$, given another factorization $T=S^{\prime} \circ h_{E}$ with $S^{\prime} \in \mathcal{L}\left(C\left(B_{E^{*}}\right), F\right)$, is $S^{\prime}$ necessarily weakly compact? The following example shows that the answer is negative.

Example 3.8. Given $T \in \mathcal{W}\left(\ell_{\infty}, \ell_{\infty}\right)=\mathcal{S I}_{\infty}^{\mathrm{wk}}\left(\ell_{\infty}, \ell_{\infty}\right)$, we shall find a factorization of $T$ through $C\left(B_{\ell_{\infty}^{*}}\right)$ in the form $T=S \circ h_{\ell_{\infty}}$ where $S: C\left(B_{\ell_{\infty}^{*}}\right) \rightarrow \ell_{\infty}$ is not weakly compact.

We first recall a couple of facts. Given a Banach space $X$, choose $e \in X$ and $\varphi \in X^{*}$ such that $\varphi(e)=1$. Denoting by $X \widehat{\otimes}_{\epsilon, \mathrm{S}} X$ the 2 -fold completed symmetric injective tensor product of $X$, the operator

$$
i: X \longrightarrow X \widehat{\otimes}_{\epsilon, \mathrm{s}} X
$$

given by

$$
\begin{aligned}
i(x) & =e \otimes x+x \otimes e-\varphi(x) e \otimes e \\
& =(e+x) \otimes(e+x)-x \otimes x-[1+\varphi(x)] e \otimes e \quad \text { for all } x \in X
\end{aligned}
$$

is an embedding onto a complemented copy of $X$ in $X \widehat{\otimes}_{\epsilon, \mathrm{s}} X$ (see [2, Theorem 3] and [1, 3.5]).

On the other hand, the operator $j: X \widehat{\otimes}_{\epsilon, \mathrm{s}} X \rightarrow C\left(B_{X^{*}}\right)$ given by

$$
j\left(\sum_{i=1}^{n} \lambda_{i} x_{i} \otimes x_{i}\right):=\sum_{i=1}^{n} \lambda_{i} h_{X}\left(x_{i}\right)^{2} \quad \text { for } \lambda_{i} \in \mathbb{K} \text { and } x_{i} \in X(1 \leq i \leq n)
$$


is an isometric embedding. If $X$ is a $C(K)$ space, the range of $j$ is complemented in $C\left(B_{X^{*}}\right)$ [21, Theorem 2.1].

For $x \in X$, we have

$$
j \circ i(x)=h_{X}(e+x)^{2}-h_{X}(x)^{2}-[1+\varphi(x)] h_{X}(e)^{2}
$$

so, for $x^{*} \in X^{*}$,

$$
\begin{aligned}
(j \circ i(x))\left(x^{*}\right) & =x^{*}(e+x)^{2}-x^{*}(x)^{2}-[1+\varphi(x)] x^{*}(e)^{2} \\
& =2 x^{*}(e) x^{*}(x)-\varphi(x) x^{*}(e)^{2}
\end{aligned}
$$

and $j \circ i(x)$ is a 2-homogeneous (continuous) scalar-valued polynomial on $X^{*}$.

Given $T \in \mathcal{W}\left(\ell_{\infty}, \ell_{\infty}\right)=\mathcal{S I}_{\infty}^{\mathrm{wk}}\left(\ell_{\infty}, \ell_{\infty}\right)$, let $k$ be the natural embedding of $h_{\ell_{\infty}}\left(\ell_{\infty}\right) \oplus j \circ i\left(\ell_{\infty}\right)$ as a subspace of $C\left(B_{\ell_{\infty}^{*}}\right)$. Define

$$
U: h_{\ell_{\infty}}\left(\ell_{\infty}\right) \oplus j \circ i\left(\ell_{\infty}\right) \longrightarrow \ell_{\infty}
$$

by

$$
U\left(h_{\ell_{\infty}}(x), j \circ i(y)\right):=T(x)+y \quad \text { for } x, y \in \ell_{\infty} .
$$

Then, $T=U \circ h_{\ell_{\infty}}$. By the injectivity of $\ell_{\infty}$ [9, Theorem 4.14], $U$ has an extension $S$ to $C\left(B_{\ell_{\infty}^{*}}\right)$ and the following diagram commutes:

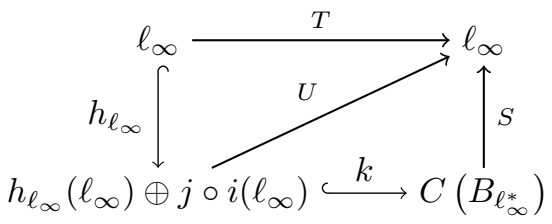

Since $U$ is not weakly compact, $S$ is not either.

In the following theorem we show that the inclusion $\mathcal{L}_{\text {ir }}(E, F) \subseteq \mathcal{I}_{\infty}(E, F)$ is strict, and obtain several characterizations of the operators in $\mathcal{I}_{\infty}(E, F)$.

Theorem 3.9. Given an operator $T \in \mathcal{L}(E, F)$, consider the following assertions:

(a) $T \in \mathcal{L}_{\text {ir }}(E, F)$;

(b) $k_{F} \circ T$ is extendible;

(c) $k_{F} \circ T$ factors through an $L_{\infty}(\Omega, \mu)$-space;

(d) $T \in \mathcal{I}_{\infty}(E, F)$;

(e) $k_{F} \circ T \in \mathcal{L}_{\text {ir }}\left(E, F^{* *}\right)$;

(f) $T^{* *}$ is extendible.

Then $(\mathrm{a}) \Rightarrow(\mathrm{b}) \Leftrightarrow(\mathrm{c}) \Leftrightarrow(\mathrm{d}) \Leftrightarrow(\mathrm{e}) \Leftrightarrow(\mathrm{f})$, but $(\mathrm{b})$ does not imply (a).

Proof. (a) $\Rightarrow$ (b). Let $i: E \hookrightarrow X$ be an embedding. By (a), $T$ admits a factorization as in diagram (2.1). Since $C\left(B_{E^{*}}\right)^{* *}$ is injective, the operator $k_{C\left(B_{E^{*}}\right)} \circ h_{E}$ has an extension $\overline{k_{C\left(B_{E^{*}}\right)} \circ h_{E}}$ to $X$, so the following diagram commutes:

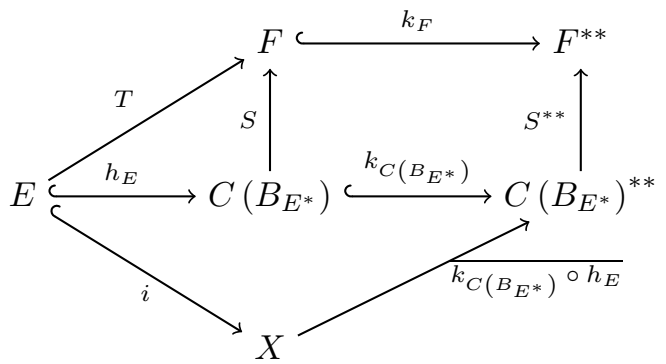


(b) $\Rightarrow$ (c) by Proposition 3.7

(c) $\Rightarrow$ (d) by the definition of the ideal $\mathcal{I}_{\infty}$ [9, page 95$]$.

(d) $\Rightarrow$ (e) is obvious.

$(\mathrm{e}) \Rightarrow$ (f). If $k_{F} \circ T \in \mathcal{L}_{\text {ir }}\left(E, F^{* *}\right)$, there is an operator $S: C\left(B_{E^{*}}\right) \rightarrow F^{* *}$ such that $k_{F} \circ T=S \circ h_{E}$. Taking second adjoints, we obtain the following commutative diagram:

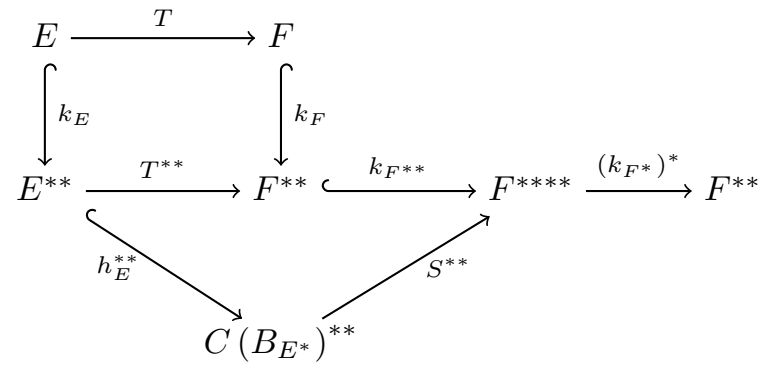

which shows that

$$
T^{* *}=\left(k_{F^{*}}\right)^{*} \circ k_{F^{* *}} \circ T^{* *}
$$

factors through the injective space $C\left(B_{E^{*}}\right)^{* *}$. Therefore, $T^{* *}$ is extendible.

(f) $\Rightarrow$ (b). If $T^{* *}$ is extendible, then it factors through an $L_{\infty}(\Omega, \mu)$-space. So $k_{F} \circ T=T^{* *} \circ k_{E}$ also factors through $L_{\infty}(\Omega, \mu)$ and is extendible.

To prove that (c) does not imply (a), let $E$ be the Bourgain-Delbaen $\mathcal{L}_{\infty}$-space with the Schur property [3, III.3]. There are embeddings $j: \ell_{1} \hookrightarrow E$ and $i: \ell_{1} \hookrightarrow$ $\ell_{\infty}$. Since $E^{* *}$ is injective [3, Proposition 1.33], $k_{E} \circ j$ admits an extension to $\ell_{\infty}$. Hence the following diagram commutes:

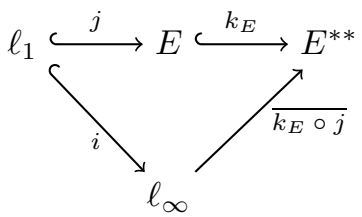

Then $k_{E} \circ j$ satisfies (c). However, $j$ does not admit an integral representation since every operator from a $C(K)$ space into $E$ is (weakly) compact.

\section{ACKNOWLEDGEMENT}

The authors are grateful to the referee for suggestions that improved the presentation of the results.

\section{REFERENCES}

[1] José M. Ansemil and Klaus Floret, The symmetric tensor product of a direct sum of locally convex spaces, Studia Math. 129 (1998), no. 3, 285-295. MR1609655

[2] Fernando Blasco, Complementation in spaces of symmetric tensor products and polynomials, Studia Math. 123 (1997), no. 2, 165-173. MR1439028

[3] Jean Bourgain, New classes of $\mathcal{L}^{p}$-spaces, Lecture Notes in Mathematics, vol. 889, SpringerVerlag, Berlin-New York, 1981. MR639014

[4] Daniel Carando, Extendible polynomials on Banach spaces, J. Math. Anal. Appl. 233 (1999), no. 1, 359-372, DOI 10.1006/jmaa.1999.6319. MR.1684392 
[5] Carmen Silvia Cardassi, Strictly p-integral and p-nuclear operators (English, with French summary), Analyse harmonique: Groupe de Travail sur les Espaces de Banach Invariants par Translation, Publ. Math. Orsay, vol. 89, Univ. Paris XI, Orsay, 1989, pp. Exp. No. 2, 22. MR.1026052

[6] W. J. Davis, T. Figiel, W. B. Johnson, and A. Pełczyński, Factoring weakly compact operators, J. Functional Analysis 17 (1974), 311-327. MR0355536

[7] Andreas Defant and Klaus Floret, Tensor norms and operator ideals, North-Holland Mathematics Studies, vol. 176, North-Holland Publishing Co., Amsterdam, 1993. MR 1209438

[8] Joseph Diestel, Sequences and series in Banach spaces, Graduate Texts in Mathematics, vol. 92, Springer-Verlag, New York, 1984. MR737004

[9] Joe Diestel, Hans Jarchow, and Andrew Tonge, Absolutely summing operators, Cambridge Studies in Advanced Mathematics, vol. 43, Cambridge University Press, Cambridge, 1995. MR 1342297

[10] J. Diestel and J. J. Uhl Jr., Vector measures, American Mathematical Society, Providence, R.I., 1977. With a foreword by B. J. Pettis; Mathematical Surveys, No. 15. MR0453964

[11] Nelson Dunford and Jacob T. Schwartz, Linear operators. Part I, Wiley Classics Library, John Wiley \& Sons, Inc., New York, 1988. General theory; With the assistance of William G. Bade and Robert G. Bartle; Reprint of the 1958 original; A Wiley-Interscience Publication. MR:1009162

[12] Manuel González and Antonio Martínez-Abejón, Tauberian operators, Operator Theory: Advances and Applications, vol. 194, Birkhäuser Verlag, Basel, 2010. MR2574170

[13] Hans Jarchow and Urs Matter, On weakly compact operators on $\mathcal{C}(K)$-spaces, Banach spaces (Columbia, Mo., 1984), Lecture Notes in Math., vol. 1166, Springer, Berlin, 1985, pp. 80-88, DOI 10.1007/BFb0074696. MR827762

[14] Joram Lindenstrauss, Extension of compact operators, Mem. Amer. Math. Soc. No. 48 (1964), 112. MR0179580

[15] Robert E. Megginson, An introduction to Banach space theory, Graduate Texts in Mathematics, vol. 183, Springer-Verlag, New York, 1998. MR1650235

[16] Constantin P. Niculescu, Absolute continuity in Banach space theory, Rev. Roumaine Math. Pures Appl. 24 (1979), no. 3, 413-422. MR.542855

[17] A. Pełczyński, Banach spaces on which every unconditionally converging operator is weakly compact, Bull. Acad. Polon. Sci. Sér. Sci. Math. Astronom. Phys. 10 (1962), 641-648. MR.0149295

[18] A. Pełczyński, On weakly compact polynomial operators on B-spaces with Dunford-Pettis property, Bull. Acad. Polon. Sci. Sér. Sci. Math. Astronom. Phys. 11 (1963), 371-378. MR 0161160

[19] Albrecht Pietsch, Operator ideals, North-Holland Mathematical Library, vol. 20, NorthHolland Publishing Co., Amsterdam-New York, 1980. Translated from German by the author. MR.582655

[20] Raymond A. Ryan, Introduction to tensor products of Banach spaces, Springer Monographs in Mathematics, Springer-Verlag London, Ltd., London, 2002. MR.1888309

[21] Ignacio Villanueva, Remarks on a theorem of Taskinen on spaces of continuous functions, Math. Nachr. 250 (2003), 98-103, DOI 10.1002/mana.200310024. MR1956604

[22] M. Zippin, Extension of bounded linear operators, Handbook of the geometry of Banach spaces, Vol. 2, North-Holland, Amsterdam, 2003, pp. 1703-1741, DOI 10.1016/S18745849(03)80047-5. MR1999607

Dipartimento di Matematica, Università di Catania, Viale Andrea Doria 6, 95125 CAtania, Italy

E-mail address: cilia@dmi.unict.it

Departamento de Matemáticas del Área Industrial, ets de Ingenieros Industriales, Universidad Politécnica de Madrid, C. José Gutiérrez Abascal 2, 28006 Madrid, Spain

E-mail address: jgutierrez@etsii.upm.es 